\title{
A comparison of idiopathic pulmonary fibrosis and chronic hypersensitivity pneumonia in terms of anterior mediastinal fat properties
}

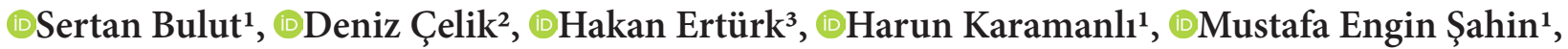 \\ (1) Özlem Sönmez ${ }^{1}$, (DÇiğdem Biber ${ }^{1}$
}

${ }^{1}$ University of Health Sciences, Atatürk Chest Diseases and Thoracic Surgery Education and Research Hospital, Department of Pulmonology, Ankara, Turkey ${ }^{2}$ Alanya Alaaddin Keykubat University, Medical Faculty, Department of Pulmonology, Antalya Turkey

${ }^{3}$ University of Health Sciences Atatürk Chest Diseases and Thoracic Surgery Education and Research Hospital, Department of Radiology, Ankara, Turkey

Cite this article as: Bulut S, Çelik D, Ertürk $\mathrm{H}$, et al. A comparison of idiopathic pulmonary fibrosis and chronic hypersensitivity pneumonia in terms of anterior mediastinal fat properties. J Health Sci Med 2022; 5(1): 262-268.

\begin{abstract}
Aim: The shape and physical properties of the anterior mediastinum can be easily affected by inflammatory lung diseases, tuberculosis, empyema, radiotherapy, chronic fibrotic lung diseases, previous surgery, and after steroid therapy. We planned to compare the properties of anterior mediastinal fat (AMF) in 3 different groups: patients with idiopathic pulmonary fibrosis (IPF), patients with chronic hypersensitivity pneumonia (cHP), and in the healthy control group. We investigate the AMF shape, dimensions, and AMF area properties on the images of high-resolution computed tomography (HRCT) and to find any difference between IPF and cHP patients in terms of AMF.

Material and Method: The study comprises a total of 80 cases in the three groups. The first group comprises 26 cases diagnosed as IPF. The second group comprises 19 cases diagnosed as cHP. The third group comprises 35 control patients. The clinical, demographical, and AMF characteristics on HRCT were retrospectively evaluated. The AMF shape and area characteristics were compared between the three groups. Results: There was no statistical difference between the mean ages of cases, BMIs, and smoking status in IPF, cHP, and control groups. Gender distribution was found statistically significant between the 3 groups $(\mathrm{p}=0.001)$. A statistically significant difference was observed between the IPF and cHP groups in terms of FVC levels $(2.67 \pm 0.59,2.14 \pm 0.80$, respectively; $p=0.024)$. Also, a statistically significant difference was observed between the IPF and cHP groups in terms of DLCO levels $(57.42 \pm 17.21 ; 77.31 \pm 35.21$; respectively; $\mathrm{p}=0.016)$. In the evaluation of AMF shape properties between two groups ( $\mathrm{cHP}$ and IPF), the concave figure was significantly more frequent in $\mathrm{cHP}$ group ( $\mathrm{p}=0.014$ ). The AMF area analyses revealed that the IPF group's areas were significantly greater than the cHP and control group's. The AP dimension of AMF analyses revealed that only the cHP group's dimensions were significantly smaller than the control group's ( $\mathrm{p}=0.037$ ). In the analysis of the transverse dimension of AMF, the IPF group's dimensions were significantly greater than the cHP and control group's ( $\mathrm{p}<0.0001$ and $\mathrm{p}=0.007$; respectively) and also the cHP group's dimensions were significantly greater than the control group's $(\mathrm{p}<0.0001)$.

Conclusion: The transverse length, total AMF area, and shape characteristics of AMF can be evaluated as a radiological marker for differential diagnosis of IPF and cHP, whose differential diagnosis may be difficult. Both the transverse length and AMF area can take greater values in the IPF group than in the cHP group.
\end{abstract}

Keywords: Idiopathic pulmonary fibrosis, chronic hypersensitivity pneumonia, anterior mediastinal fat

\section{INTRODUCTION}

Interstitial lung diseases (ILDs) are chronic lung diseases with similar clinical, radiological and pulmonary function test results (1). Although IPF is one of the most common forms of ILD, its origin is still uncertain and it has a poor prognosis $(1,2)$. Although the incidence of IPF varies from region to region, it is incidence is 0.09-0.93 in 10000 (in ten thousand) and its prevalence varies between $0.33-4.51$ in 10000 (in ten thousand) (3). The 5 -year survival of IPF is $40 \%$ (4). The diagnosis of IPF is a result of the algorithm in which the HRCT and Histopathology patterns are evaluated together (5) and therefore HRCT findings are very important in the diagnosis and differential diagnosis of IPF $(5,6)$. Chronic hypersensitivity pneumonia is an inflammatory and/ or fibrotic disease that involves the lung parenchyma and small airways and frequently interferes with IPF (7). Environmental and occupational exposures may be associated with IPF as well as with CHP (8).

The mediastinum is the anatomical space located in the thoracic cavity, which is limited by the sternum anteriorly, the pleura laterally, the vertebrae posteriorly, and the thoracic inlet superiorly. The mediastinum is parted into 4 anatomical compartments; anterior, middle, posterior, 
and upper mediastinum (9). The anterior mediastinum has a structure that can be affected by various pathological conditions and easily adapt to these changes (10). The shape and physical properties of the anterior mediastinum can be easily affected by inflammatory lung diseases, tuberculosis, empyema, radiotherapy, chronic fibrotic lung diseases, previous surgery, and after steroid therapy (11-13). There are studies in which the fat ratio in the body is evaluated (the skeletal muscle index) in diagnostic processes (14).

In 2014, Hassan and Abo-Elhamd (15) investigated the properties of anterior mediastinal fat (AMF) in patients with idiopathic pulmonary fibrosis (IPF), and in 2006, Lee et al. (11) investigated the properties of AMF in patients with IPF and nonspecific interstitial pneumonia (NSIP). In 2020, González et al. (16) examined the effect of AMF on prognosis in pulmonary fibrosis patients who underwent lung transplantation. In this study, we planned to compare the properties of AMF in the patient 3 groups: patients with IPF, patients with chronic hypersensitivity pneumonia (cHP), and in the healthy control group for the first time.

\section{MATERIAL AND METHOD}

The study was carried out with the permission of University of Health Sciences Atatürk Education and Training Hospital Clinical Researchs Ethics Committee (Date: 12/10/2021, Decision No: 2012-KAEK-15/2390). All procedures were performed adhered to the ethical rules and principles of the Helsinki Declaration.

\section{Patients}

Patients who were evaluated and diagnosed in the 'diffuse parenchymal lung diseases multidisciplinary council' of our institution enrolled in this study. Our institution is a tertiary education and research hospital and specializes in pulmonary diseases and chest surgery. The study comprises 3 patient groups. The first group comprises 26 cases that were evaluated according to ATS/ERS criteria (5). and subsequently diagnosed as IPF. The second group comprises 19 cases who were evaluated and finally diagnosed as chronic hypersensitivity pneumonia (cHP) in the light of current guidelines (17). The third group comprises 35 control patients without any disease.

The cases included in our study are those diagnosed with IPF and cHP among all patients in the last 8 years. A total of 80 cases in the three groups were evaluated. There was no known past and/or active inflammatory or infectious disease with patients and control groups. None of the patients in the IPF and cHP groups received corticosteroids before high-resolution chest tomography (HRCT) evaluation. None of the cases had diabetes, atherosclerotic cardiovascular disease, and had no history of thoracic surgery and radiotherapy. The demographic data, BMI, smoking status, hemogram, and biochemistry analysis results of these cases were recorded retrospectively. Shape features, anteroposterior and transverse lengths, and AMF area were calculated of all cases. Pulmonary function tests (PFTs) and diffusing capacity for carbon monoxide (DLCO) results were recorded in IPF and cHP groups.

\section{High-resolution Chest Tomography (HRCT)}

All cases HRCT results analyzed. Cases were in supine position and analysis was performed without iv. contrast agents. $1.5 \mathrm{~mm}$ thick sections were taken in the axial plane with 128 multidetector CT device (Ingenuity CT, Philips Healthcare, Andover, MA, USA). Display parameters were $\mathrm{Kv}: 120$; $\mathrm{mA}: 160$, rotation time:0,5 s; collimation:64x0,625; FOV: $220 \mathrm{~mm}$. The window settings were determined as $\mathrm{W}: 350, \mathrm{C}: 60$ for mediastinum and W:-1600, C:-600 for parenchyma.

The shape of the anterior mediastinal fat was categorized as concave, flat, or convex at the level of the main pulmonary trunk. The anteroposterior (AP) (from the posterior wall of the sternum to the anterior wall of the ascending aorta) and transverse dimensions (the width of the posterior wall of the sternum in contact with the anterior mediastinal fat) at the level of the main pulmonary trunk was also measured (15) and the AMF area was determined manually and calculated with the CT device in $\mathrm{cm}^{2}$.

\section{Statistical Analysis}

All the analysis was performed with SPSS System software (version 22.0). The shapes of the anterior mediastinal fat in the IPF and cHP and control groups were compared using the Chi-square test. Amounts of anterior mediastinal fat, the retrosternal AP, transverse dimensions of the anterior mediastinum, the weights, and body mass indexes (BMIs) were compared using one-way analysis of variance (ANOVA). Correlations between forced vital capacity (FVC), forced expiratory volume in the first second (FEV1) and DLCO with AP and transverse diameters and areas of anterior mediastinal fat were performed using Pearson's correlation coefficient. AUC was calculated by performing ROC analysis of AMF area, AP length, and transverse dimension values, which are our qualitative measurements for the diagnosis of IPF. Sensitivity and specificity values were determined for the statistically significant transverse dimension and AMF area values, and cut-off values were determined. Multivariate regression analyzes were performed by age, gender, smoking status, and BMIs separately, which are likely to be confounding factors for AMF shape features, length, and AMF area measurements. The fit of the models created in this regression analysis was evaluated with the Hosmer and Lemeshow test, and a p-value above 0.05 was considered a good fit. Hemogram, NLR, CRP, and albumin values were analyzed to evaluate whether 


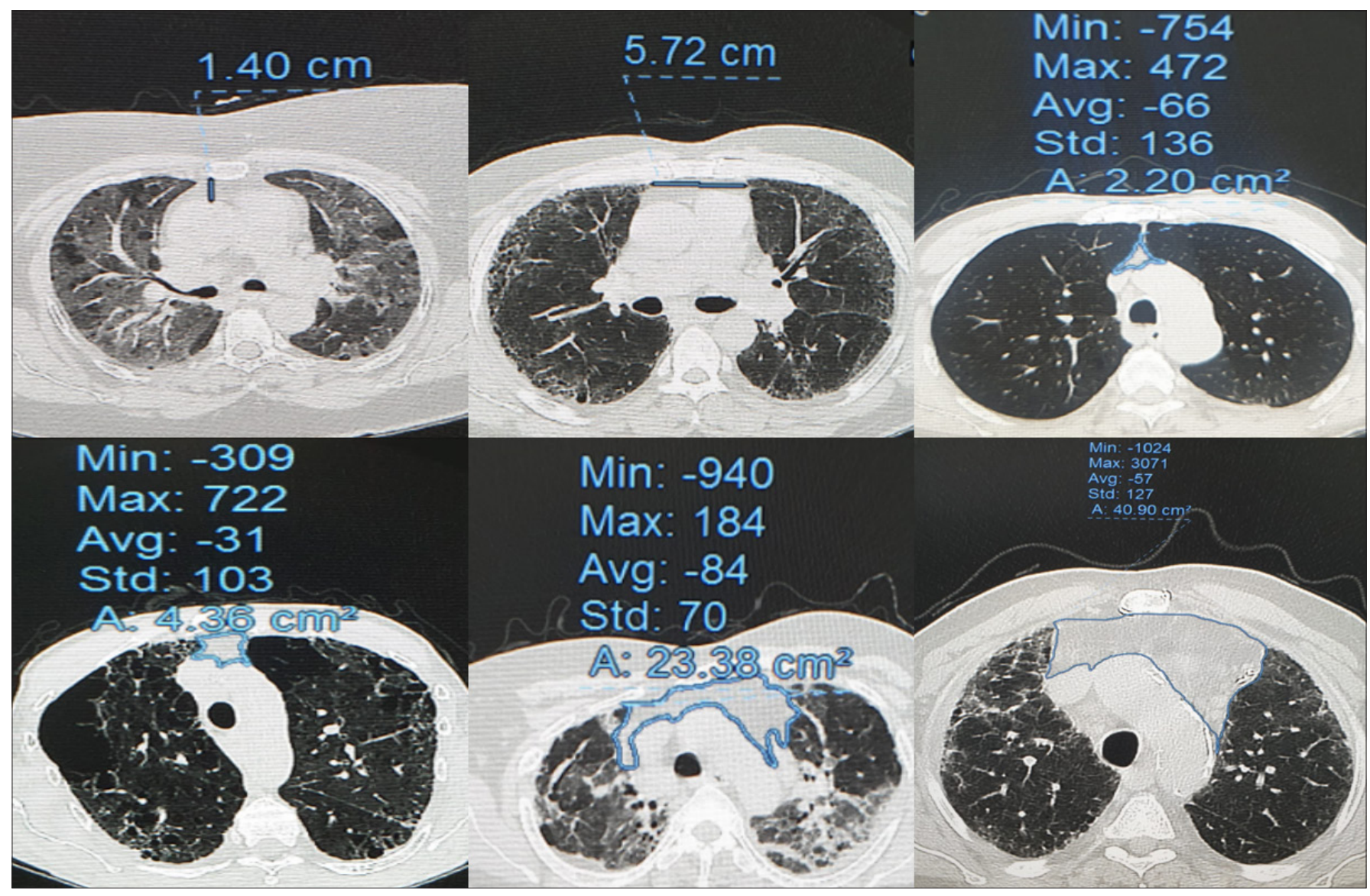

Picture 1. A. The anteroposterior (AP) (from the posterior wall of the sternum to the anterior wall of the ascending aorta), B. Transverse dimensions (the width of the posterior wall of the sternum in contact with the anterior mediastinal fat) at the level of the main pulmonary trunk, C. Concave shape, D. Flat shape, $\mathbf{E}$ and F: convex shape, ${ }^{\star}$ All AMF areas were determined manually and calculated with the $\mathrm{CT}$ device in $\mathrm{cm}^{2}$.

there was a relationship between AMF and systemic inflammation. In addition, IPF and cHP patient groups were compared in terms of PFTs and DLCO values. The p-value less than 0.05 was considered to indicate statistically significant differences.

\section{RESULTS}

Demographic data of three groups were compared. Gender distribution was 24 males 2 females in the IPF group, 5 males 14 females in the cHP group, and 30 males and 5 females in the control group. Gender distribution was found statistically significant between the 3 groups $(\mathrm{p}=0.001$ ). There was no statistical difference between the mean ages of cases in IPF, cHP, and control groups $(67.50 \pm 12,67.50 \pm 12$, and 67.68 \pm 8.30 , respectively) $(\mathrm{p}=0.67)$ (Table $\mathbf{1})$.
BMI mean value was $28.22 \mathrm{~kg} / \mathrm{m} 2(3.56 \%)$ in the IPF group, $29.97 \mathrm{~kg} / \mathrm{m} 2(5.99 \%)$ in the cHP group and $28.92 \mathrm{~kg} / \mathrm{m} 2(3.29 \%)$ in the control group. There was no statistical difference between the three groups by the means of BMIs ( $\mathrm{p}=0.380$ ). There was no correlation between the smoking status of the three groups $(\mathrm{p}=0.054)$.

The AP dimension of AMF analyses revealed that only the cHP group's dimensions were significantly smaller than the control group's $(p=0.037)$. In the analysis of the transverse dimension of AMF, the IPF group's dimensions were significantly greater than the control group's $(\mathrm{p}<0.0001)$ and greater than the cHP group's $(\mathrm{p}=0.007)$ and also the cHP group's dimensions were significantly greater than the control group's ( $\mathrm{p}<0.0001)$.

\begin{tabular}{|lcccc|}
\hline Table 1. Demographic and clinical characteristics & & & \\
\hline Characteristic & IPF group $(\mathbf{n = 2 6})$ & cHP group $(\mathbf{n = 1 9 )}$ & Control group (n=35) & p value \\
\hline Age (years) (mean \pm SD) & $67.50 \pm 12$ & $61.52 \pm 12.75$ & $67.68 \pm 8.30$ & 0.067 \\
Male gender (n) (\%) & $24(92.3 \%)$ & $5(26.31 \%)$ & $30(85.71 \%)$ & $0.001^{*}$ \\
BMI mean \pm sd (kg/m2) & $28.22(3.56 \%)$ & $29.97(5.99 \%)$ & $28.92(3.29 \%)$ & 0.380 \\
Smoking status (n) (\%) & & & & 0.054 \\
Current & $1(3.84 \%)$ & $1(5.26 \%)$ & $5(14.28 \%)$ \\
Former & $25(96.15 \%)$ & $16(84.21 \%)$ & $23(65.71 \%)$ \\
Never & $0(0 \%)$ & $2(10.52 \%)$ & $7(20.00 \%)$ \\
\hline
\end{tabular}

Note. ${ }^{*} \mathrm{p}<0.05$ BMI: Body mass index 
The AMF area analyses revealed that the IPF group's areas were significantly greater than the control group's areas $(\mathrm{p}<0.0001)$ and greater than the $\mathrm{cHP}$ group's areas $(p=0.0037)$ and also the cHP group's areas were significantly greater than the control group's $(\mathrm{p}=0.008)$ (Figure 1, 2 and Table 2).

The AMF shape features evaluation showed a concave figure in 4 cases with cHP and 17 cases of the control group ( $\mathrm{p}<0.0001$ ), a flat figure in 3 cases IPF, 1 case with

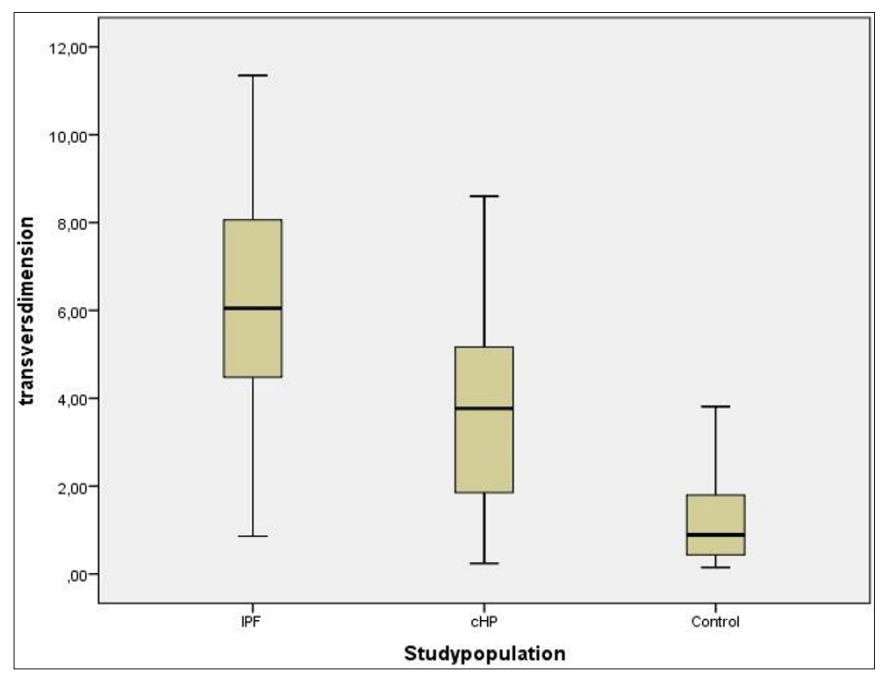

Figure 1. The IPF group's transverse dimensions were significantly greater than the control group's $(\mathrm{p}<0.0001)$ and greater than the $\mathrm{cHP}$ group's $(p=0.007)$ and also the cHP group's transverse dimensions were significantly greater than the control group's $(\mathrm{p}<0.0001)$.

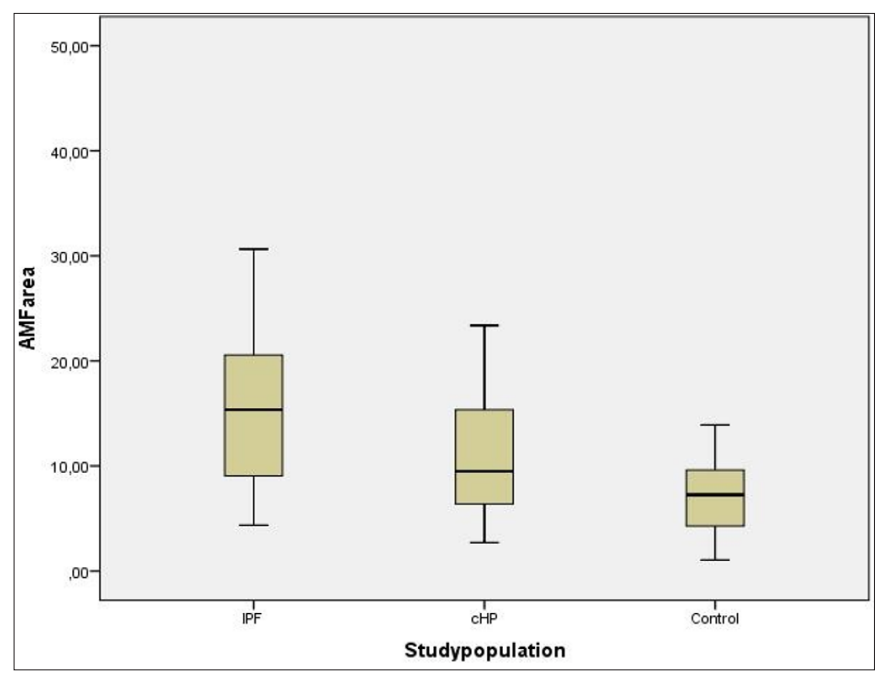

Figure 2. The IPF group's AMF areas were significantly greater than the control group's AMF areas $(\mathrm{p}<0.0001)$ and greater than the cHP group's AMF areas $(\mathrm{p}=0.0037)$ and also the $\mathrm{cHP}$ group's $\mathrm{AMF}$ areas were significantly greater than the control group's ( $\mathrm{p}=0.0008)$.
cHP, and 15 cases of the control group ( $\mathrm{p}=0.002)$, convex figure in 23 cases with IPF, 14 cases with cHP and 3 cases of the control group $(p<0.0001)$ (Table 3$)$. In the evaluation of AMF shape properties between two groups (cHP and IPF), the concave figure was significantly more frequent in cHP group $(\mathrm{p}=0.014)$ (Table 4).

Regression analyzes of AMF shape features, lengths, and area measurements were performed in a model in which confounding risk factors such as age, BMI, smoking, and gender were included. The fit of all models created in this regression analysis was evaluated with the Hosmer and Lemeshow test. It was seen that all of them had a good model fit ( $p>0.05$ ). In these regression models, none of the possible confounding factors were at the level of statistical significance $(p>0.05)$. For example, in the evaluation of the convex shape in terms of confounding factors in Table 5, the Hosmer and Lemeshow test result indicated a good model fit with $\mathrm{p}=0.912$, while there was no statistical significance in the risk factors examined ( $p>0.05)$.

Table 3. Different shapes of anterior mediastinum in the three groups

\begin{tabular}{|lcccc|}
\hline AMP shape & Normal n (\%) & cHP n (\%) & IPF n (\%) & p-value \\
\hline Concave & $17(48.6 \%)$ & $4(21 \%)$ & $0(0 \%)$ & $0.000^{*}$ \\
Flat & $15(42.9 \%)$ & $1(5.3 \%)$ & $3(11.2 \%)$ & $0.002^{*}$ \\
Convex & $3(8.6 \%)$ & $14(73.7 \%)$ & $23(88.4 \%)$ & $0.000^{*}$ \\
Note. ${ }^{*}$ p-value $<0.05$; the statistical result was obtained by the Chi-square test.
\end{tabular}

Table 4. Different shapes of anterior mediastinum in the two groups

\begin{tabular}{|llll|}
\hline AMP Shape & cHP n (\%) & IPF $\mathbf{n}(\%)$ & p-value $\mathbf{n}(\%)$ \\
\hline Concave & $4(21 \%)$ & $0(0.0 \%)$ & $0.014^{*}$ \\
Flat & $1(5.3 \%)$ & $3(11.2 \%)$ & 0.465 \\
Convex & $14(73.7 \%)$ & $23(88.4 \%)$ & 0.2 \\
\hline \multicolumn{4}{|l}{ Note. ${ }^{*}$ p-value $<0.05$; Statistical result was obtained by the Chi-square test } \\
\hline
\end{tabular}

Table 5. The evaluation of the convex shape in terms of confounding factors with multivariate logistic regression analyze

\begin{tabular}{|lcc|}
\hline Risk factors & OR (\%95 CI) & p-value \\
\hline Age & $0.978(0.932-1.027)$ & 0.369 \\
Gender & $1.882(0.594-5.959)$ & 0.282 \\
Smoking status & $2.203(0.230-21.088)$ & 0.493 \\
BMI & $0.951(0.839-1.077)$ & 0.425 \\
\hline
\end{tabular}

The Hosmer and Lemeshow test result indicated a good model fit with $\mathrm{p}=0.912$ and Chi square $=3.333$. There was no statistical significance in the risk factors examined $(\mathrm{p}>0.05)$.

\begin{tabular}{|c|c|c|c|c|c|c|c|}
\hline Variables & $\begin{array}{c}\text { IPF } \\
(\text { mean } \pm S D)\end{array}$ & $\begin{array}{c}\text { cHP } \\
(\text { mean } \pm S D)\end{array}$ & $\begin{array}{c}\text { Control } \\
(\text { mean } \pm S D)\end{array}$ & $\begin{array}{l}\text { Normal } \\
\text { vs cHP }\end{array}$ & $\begin{array}{c}\text { Normal } \\
\text { vs IPF }\end{array}$ & $\begin{array}{c}\text { cHP } \\
\text { vs IPF }\end{array}$ & $\begin{array}{l}\text { Normal vs } \\
\text { cHP vs IPF }\end{array}$ \\
\hline Body mass index $(\mathrm{kg} / \mathrm{m} 2)$ & $28.22 \pm 3.56$ & $29.97 \pm 5.99$ & $28.92 \pm 3.29$ & 0.406 & 0.431 & 0.226 & 0.380 \\
\hline AP dimension $(\mathrm{cm})$ & $2.27 \pm 0.93$ & $1.81 \pm 0.82$ & $2.33 \pm 0.85$ & $0.037^{\star}$ & 0.797 & 0.096 & 0.809 \\
\hline Transverse dimension $(\mathrm{cm})$ & $6.10 \pm 2.67$ & $3.84 \pm 2.60$ & $1.28 \pm 1.15$ & $0.000^{\star}$ & $0.000^{*}$ & $0.007^{*}$ & $0.001^{*}$ \\
\hline Area of AMF $(\mathrm{cm} 2)$ & $15.94 \pm 8.44$ & $11.01 \pm 6.16$ & $7.34 \pm 3.66$ & $0.008^{*}$ & $0.000^{*}$ & $0.037^{*}$ & $0.001^{*}$ \\
\hline
\end{tabular}

Note. ${ }^{*} \mathrm{p}$ value $<0.05$; Data are presented as means SDs. 
Correlation between the changes in the diameters of AMF with PFTs (FEV1, FVC, and DLCO) in IPF and cHP groups revealed a significant correlation between AP dimension and FEV 1 and FVC $(r=502, p<0.0001$ and $\mathrm{r}=481, \mathrm{p}=0.001$, respectively).

As seen in Figure 3, ROC curves and AUC were compared between AMF area, AP length, and transverse dimension values to predict IPF diagnosis. Transverse dimension showed the best $\mathrm{AUC}=0.86$, (CI 95\% 0.7830.951, $\mathrm{p}<0.0001)$, followed by the AMF area with an AUC $=0.77$ (CI95\%, 0.661-0.886, p<0.0001), while AP length were not significant $(\mathrm{p}=0.551)$. The Transverse dimension sensitivity was $80 \%$, specificity was $77 \%$ cutoff $=3.79 \mathrm{~cm}$ in the diagnosis of IPF. The AMF area sensitivity was $73 \%$, specificity was $72 \%$ cut off $=9.82 \mathrm{~cm}^{2}$ in the diagnosis of IPF.

Systemic inflammatory biomarkers were evaluated to determine the relationship between AMF and systemic inflammation. No relationship was found between AMF and inflammatory biomarkers, such as neutrophil, lymphocyte, neutrophil/lymphocyte ratio (NLR), CRP, and albumin levels. When the 3 groups were compared, there was a significant difference in white blood cell (WBC) levels $(\mathrm{p}=0.028)$, while no significant difference was observed between IPF and control groups in the subgroup analysis $(9.68 \pm 2.99,9.11 \pm 2.90$, respectively; $\mathrm{p}=0.455)$. Also, there was no significant difference between $\mathrm{cHP}$ and the control group $(7.84 \pm 2.46,9.11 \pm 2.90$, respectively; $\mathrm{p}=0.055$ ).

PFTs could only be evaluated in the IPF and cHP groups, but not in the control group. A statistically significant difference was observed between the IPF and cHP groups in terms of FVC levels $(2.67 \pm 0.59,2.14 \pm 0.80$, respectively; $\mathrm{p}=0.016)$ and FEV1 levels $(2.22 \pm 0.44,1.75 \pm 0.65$, respectively; $\mathrm{p}=0.006)$. Also, a statistically significant difference was observed between the IPF and cHP groups in terms of DLCO levels (57.42 \pm 17.21 ; 77.31 \pm 35.21 ; respectively; $\mathrm{p}=0.016$ ) (Table 6).

\section{DISCUSSION}

This is the first study in which the properties of AMF were evaluated in cHP patients and also investigated together in CHP-IPF patients. This article aimed to determine the relationship between AMF features in patients with IPF and $\mathrm{cHP}$ and to evaluate these features as a marker.

In this study, we defined the anatomical features of AMF in IPF, cHP, and control patients with HRCT. We showed that transverse dimension and convex shape in IPF and cHP patients were statistically significantly larger and different when compared to control patients. In this context, we have shown that transverse length and convex shape can be markers.

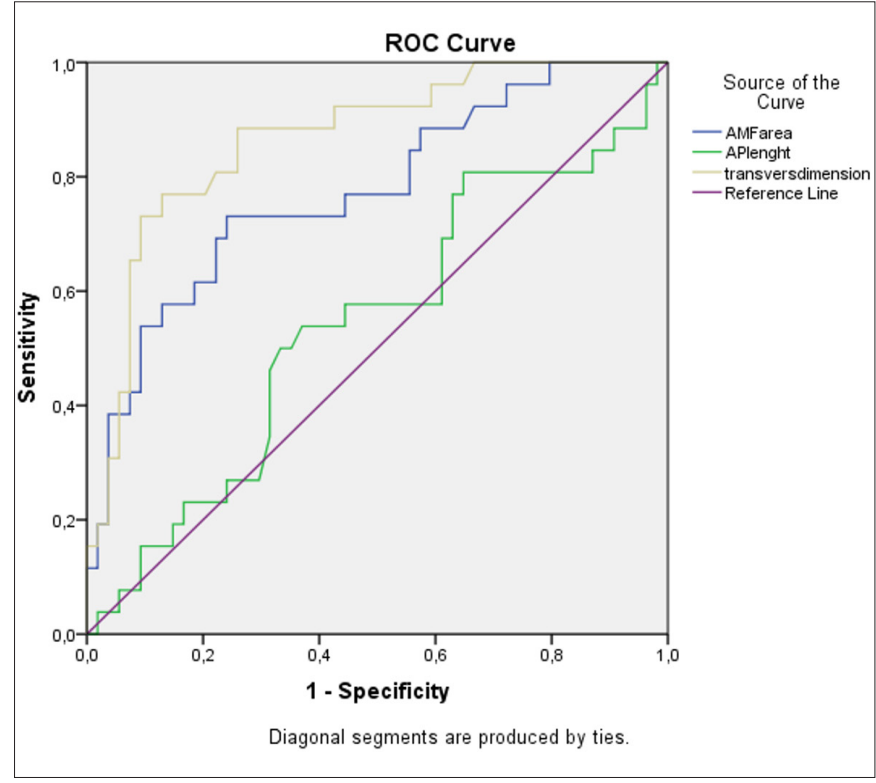

Figure 3. The ROC curves and AUC were compared between AMF area, AP length, and transverse dimension values to predict IPF diagnosis. Transverse dimension showed the best $A U C=0.86$, (CI $95 \% 0.783-0.951, \mathrm{p}<0.0001)$, followed by the AMF area with an AUC $=0.77$ (CI95\%, 0.661-0.886, $\mathrm{p}<0.0001)$, while AP length were not significant $(\mathrm{p}=0.551)$. The transverse dimension sensitivity was $80 \%$, specificity was $77 \%$ cutoff $=3.79 \mathrm{~cm}$ in the diagnosis of IPF. The AMF area sensitivity was $73 \%$, specificity was $72 \%$ cut off $=9.82 \mathrm{~cm} 2$ in the diagnosis of IPF.

Table 6. Systemic inflammation markers between three groups

\begin{tabular}{|c|c|c|}
\hline Characteristic & $\mathrm{n}=\mathbf{8 0}$ & p value \\
\hline WBC unit $\dagger($ mean \pm SD) & $9.00 \pm 2.88$ & 0.028 \\
\hline \multicolumn{3}{|l|}{ WBCS } \\
\hline IPF vs control group & & 0.455 \\
\hline cHP vs control group & & 0.055 \\
\hline Neutrophil $\dagger($ mean \pm SD) & $6.34 \pm 2.58$ & 0.123 \\
\hline Neutrophil $(\%)^{\star}($ mean \pm SD $)$ & $69.71 \pm 11.10$ & 0.736 \\
\hline Lymphocyte ${ }^{\star}($ mean $\pm S D)$ & $1.83 \pm 0.78$ & 0.113 \\
\hline Lymphocyte $(\%)^{\star}($ mean \pm SD $)$ & $21.21 \pm 8.80$ & 0.265 \\
\hline $\mathrm{NLR} \dagger($ mean $\pm \mathrm{SD})$ & $4.57 \pm 3.89$ & 0.449 \\
\hline Hemoglobin* (mean \pm SD $)$ & $13.94 \pm 2.07$ & 0.590 \\
\hline Hematocrit ${ }^{*}($ mean $\pm S D)$ & $43.26 \pm 5.88$ & 0.989 \\
\hline Platelet $^{\star}($ mean $\pm S D)$ & $25605 \pm 90510.63$ & 0.156 \\
\hline $\mathrm{CRP} \dagger($ mean $\pm \mathrm{SD})$ & $21.81 \pm 42.01$ & 0.205 \\
\hline Albumin $\dagger($ mean \pm SD $)$ & $37.11 \pm 5.66$ & 0.965 \\
\hline $\mathrm{FVC} \neq($ mean $\pm \mathrm{SD})$ & & 0.024 \\
\hline IPF & $2.67 \pm 0.59$ & \\
\hline cHP & $2.14 \pm 0.80$ & \\
\hline FEV $1 \ddagger($ mean $\pm S D)$ & & 0.006 \\
\hline IPF & $2.22 \pm 0.44$ & \\
\hline cHP & $1.75 \pm 0.65$ & \\
\hline $\mathrm{DLCO} \neq \%($ mean $\pm \mathrm{SD})$ & & 0.016 \\
\hline IPF & $57.42 \pm 17.21$ & \\
\hline cHP & $77.31 \pm 35.21$ & \\
\hline $\begin{array}{l}\text { WBC: White blood cell, NLR: Neutrop } \\
\text { FVC: Force vital capacity, DLCO: Diff } \\
\text { †Kruskal-Wallis test, „Indipendent } t-t \\
\text { analyzed for these two groups: IPF an }\end{array}$ & $\begin{array}{l}\text { hocyte ratio, CRP: C- } \\
\text { pacity for carbon mon } \\
\text { nn-Whithney U Test, }\end{array}$ & $\begin{array}{l}\text { ive protein, } \\
\text { e*ANOVA, } \\
\text { C and DLCC }\end{array}$ \\
\hline
\end{tabular}


In addition, when we compared the AMF area, we observed that the measurements of IPF patients had the highest value. For the diagnosis of IPF, we found that the values of transverse dimension and area values were statistically significant by calculating AUC by ROC analysis of AMF qualitative measurements. We determined the cut-off values of these two data as 3.79 $\mathrm{cm}$ and $9.82 \mathrm{~cm}^{2}$, respectively.

In previous studies examining the properties of AMF, the relationship between AMF and systemic inflammation was never examined $(11,15,16)$. This is the first study evaluating the properties of AMF with systemic inflammation in both IPF and cHP patients, and it has been shown that there is no relationship between AMF and systemic inflammation.

AMF characteristics can be affected by infectious and inflammatory lung diseases, thoracic surgery and thoracic radiotherapy, and steroid therapy given for any reason (10-12). Likewise, intrathoracic fat can be affected by obesity as well as diabetes and is associated with ASHH (18). We did not include the cases with the abovementioned conditions.

In previous studies, the IPF group's AMP transverse length tended to be taller, and AP length was shorter compared to the control group $(11,15)$. In our study; Transverse length and AMF area were found to be consistent with previous studies in both IPF and cHP groups, which were statistically significantly different from the control group.

We also determined a cut-off value using qualitative measures of AMF that had not been evaluated in previous studies. In the diagnosis of IPF for the transverse dimension our calculated cut-off value $=3.79$ $\mathrm{cm}$, sensitivity $80 \%$, specificity $77 \%(\mathrm{p}<0.0001)$, and for the AMF area cut-off value $=9.82 \mathrm{~cm}^{2}$, sensitivity $73 \%$, specificity $72 \%(\mathrm{p}<0.0001)$.

Lee et al. (11) found that in IPF patients, AMF tended to be transversely taller and shorter at the AP plane compared with the control group. They explained this result with the following mechanism: "The widening of the transverse dimension and the shortening of the anteroposterior dimension of the anterior mediastinal fat seem to be bilateral tensile forces induced by subpleural fibrosis of the lung tissue that was adjacent to the mediastinum.." In our study, transverse length and AMF area feature support this mechanism. In our study, the fact that AP length is statistically less than normal controls in cHP, which can also be a fibrotic disease, also supports this mechanism.

We showed that systemic inflammatory markers were similar in all 3 groups. For all these reasons, we also support this possible mechanism. However, interestingly, we cannot explain the similarity of the AP length in the
IPF and the control group, which is revealed in our study, by any mechanism.

In previous studies, the relationship between AMF and systemic inflammation was not discussed. In our study, systemic inflammatory markers were evaluated to determine the relationship between AMF and systemic inflammation. No relationship was found between AMF and inflammatory biomarkers, such as neutrophil, lymphocyte, neutrophil/lymphocyte ratio (NLR), CRP, and albumin levels.

In previous studies, AMF shape features tended to be convex in the IPF group and concave in normal healthy subjects $(11,15,16)$. In our study, it was found as concave $(p<0.0001)$, flat $(p=0.002)$, convex $(p<0.0001)$ for 3 groups. In the subgroup analysis, the $\mathrm{cHP}$ group tended to be concave in terms of AMF shape properties compared to the IPF group $(\mathrm{p}=0.014)$.

As a conclusion, we think that the transverse length, AMF area, and shape characteristics of AMF can be evaluated as a radiological marker for differential diagnosis of IPF and cHP, whose differential diagnosis may be difficult. It has been observed that both the transverse length and AMF area can take greater values in IPF patient groups than in the cHP group.

In the analysis of PFTs between the IPF and cHP groups, we found a significant difference $(2,67 \pm 0,59$; $2,14 \pm 0,80$; respectively, $\mathrm{p}=0,024)$. The $\mathrm{cHP}$ patients were symptomatic and we used their PFTs before steroid therapy. Because of this, compared to the IPF group, PFTs were significantly low and recovery was expected after steroid therapy (19). Also, a significant difference was observed between the IPF and cHP groups in terms of DLCO levels $(57,42 \pm 17,21 ; 77,31 \pm 35,21$; respectively; $\mathrm{p}=0,016$ ). This is consistent with previous literature data (20).

Hassan et al. (15) reported that AMF transverse length was negatively correlated with pulmonary functions and AP length was positively correlated in patients with IPF. In our study, we found a relationship between AP length and FVC and FEV1 ( $r=502, p<0.0001$ and $\mathrm{r}=481, \mathrm{p}=0.001$; respectively). Interestingly, there was no relationship with PFTs in terms of transverse length and AMF area in both case groups.

This study's limitations were being a retrospective singlecenter study and a relatively limited number of patients.

\section{CONCLUSION}

Qualitative and quantitative characteristics of AMF can be an additional help in distinguishing from normal healthy adults during radiological evaluation while making cHP and IPF diagnoses. In addition, quantitative 
measurements of AMF can help us to distinguish between two clinical entities such as IPF and cHP, which can sometimes be difficult to distinguish from each other. We recommend that these results could be supported by prospective studies with a higher number of cases.

\section{ETHICAL DECLARATIONS}

Ethics Committee Approval: The study was carried out with the permission of University of Health Sciences Atatürk Education and Training Hospital Clinical Research Ethics Committee (Date: 12/10/2021, Decision No: 2012-KAEK-15/2390).

Informed Consent: Because the study was designed retrospectively, no written informed consent form was obtained from patients.

Referee Evaluation Process: Externally peer-reviewed.

Conflict of Interest Statement: The authors have no conflicts of interest to declare.

Financial Disclosure: The authors declared that this study has received no financial support.

Author Contributions: All of the authors declare that they have all participated in the design, execution, and analysis of the paper, and that they have approved the final version.

\section{REFERENCES}

1. Cabrera Cesar E, Lopez-Lopez L, Lara E, et al. Serum biomarkers in differential diagnosis of idiopathic pulmonary fibrosis and connective tissue disease-associated interstitial lung disease. J Clin Med 2021; 10: 3167.

2. Caminati A, Madotto F, Conti S, Cesana G, Mantovani L, Harari $S$. The natural history of idiopathic pulmonary fibrosis in a large European population: the role of age, sex and comorbidities Intern Emerg Med 2021; 16: 1793-802.

3. Maher TM, Bendstrup E, Dron L, et al. Global incidence and prevalence of idiopathic pulmonary fibrosis. Respir Res 2021; 22: 197.

4. Ley B, Collard HR, King TE Jr. Clinical course and prediction of survival in idiopathic pulmonary fibrosis. Am J Respir Crit Care Med 2011; 183: 431-40.

5. Raghu G, Remy-Jardin M, Myers JL, et al. Diagnosis of idiopathic pulmonary fibrosis. an official ATS/ERS/JRS/ALAT clinical practice guideline. Am J Respir Crit Care Med 2018; 198: e44-e68.

6. Hobbs S, Chung JH, Leb J, Kaproth-Joslin K, Lynch DA. Practical imaging interpretation in patients suspected of having idiopathic pulmonary fibrosis: official recommendations from the Radiology Working Group of the Pulmonary Fibrosis Foundation. Radiol Cardiothorac Imaging 2021; 3: e200279.

7. Koster MA, Thomson CC, Collins BF, Jenkins AR, Ruminjo JK, Raghu G. Diagnosis of hypersensitivity pneumonitis in adults, 2020 clinical practice guideline: summary for clinicians. Ann Am Thorac Soc 2021; 18: 559-66.

8. Park Y, Ahn C, Kim TH. Occupational and environmental risk factors of idiopathic pulmonary fibrosis: a systematic review and meta-analyses. Sci Rep 2021; 11: 4318.

9. W. K. (2008). Mediastinum. In Comprehensive Cytopathology (pp. 773-809). WB Saunders.
10.Proto AV. Conventional chest radiographs: anatomic understanding of newer observations. Radiology 1992; 183: 593603.

11.Lee $\mathrm{CH}$, Lee $\mathrm{HJ}$, Son $\mathrm{KR}$, et al. CT analysis of the anterior mediastinum in idiopathic pulmonary fibrosis and nonspecific interstitial pneumonia. Korean J Radiol 2006; 7: 173-9.

12. Teates CD. Steroid-induced mediastinal lipomatosis. Radiology. 1970; 96: 501-2.

13. Toei H, Furuse M, Shinozaki T, Sohara Y. Nihon. Igaku Hoshasen Gakkai Zasshi 1997; 57: 493-498.

14. Jalaber C, Lemerre-Poincloux J, Jouneau S, et al. Usefulness of body composition CT analysis in patients with idiopathic pulmonary fibrosis: a pilot study. Acad Radiol 2021; S1076-6332(21)00328-7.

15. Hassan WA, Abo-Elhamd E. Anterior mediastinal fat changes in idiopathic pulmonary fibrosis: A preliminary study. Open J Respir Dis 2014.

16.González, F. J., Alvarez, A., Cantador, et al. Relationship among radiological measurements of anterior mediastinal fat and outcomes of lung transplantation in fibrotic patients. Archivos de Bronconeumología 2020; 56: 710-7.

17. Morisset J, Johannson KA, Jones KD, et al. Identification of diagnostic criteria for chronic hypersensitivity pneumonitis: an International Modified Delphi Survey. Am J Respir Crit Care Med 2018; 197: 1036-44.

18. Rosito GA, Massaro JM, Hoffmann U, et al. Pericardial fat, visceral abdominal fat, cardiovascular disease risk factors, and vascular calcification in a community-based sample: the Framingham Heart Study. Circulation 2008; 117: 605-13.

19.Pereira CA, Gimenez A, Kuranishi L, Storrer K. Chronic hypersensitivity pneumonitis. J Asthma Allergy 2016; 9: 171-81.

20. Morell F, Villar A, Montero MÁ, et al. Chronic hypersensitivity pneumonitis in patients diagnosed with idiopathic pulmonary fibrosis: a prospective case-cohort study. Lancet Respir Med 2013; 1: 685-94. 